\title{
Application Function and Daily Management Decision of Hotel Data Center
}

\author{
Hui LIANG ${ }^{1,2, a^{*}}$ \\ ${ }^{1}$ Hubei University of Economics, Wuhan, Hubei, China 430205 \\ ${ }^{2}$ Wuhan University, Wuhan, Hubei, China 430072 \\ àHiang@hbue.edu.cn
}

Keywords: Hotel Data Center; Application Functions; Daily Management; Decision

Abstract. For the definition of data center, there has been no a very clear concept classification yet in professional studies. From the perspective of hardware, data center is a data processing area composed by a set of facilities; from the perspective of hardware, it can be seen as a data warehouse with a large capacity. For the business management of a hotel, using high-configuration computer for establishing data center is a reform trend of information-based hotel management. In this paper, the characteristics of data center is first analyzed, the main functions of hotel data center are introduced, and also a comprehensive decision for the daily management of data center is proposed. Hopefully, this paper will be helpful for the future studies.

\section{Introduction}

Hotel consumption is a main form for expressing market management activities. Along with the arrival of the period of rapid consumption and fashion consumption, hotel consumption has changed into an indispensable part of the daily life of people. For the business management of a hotel, how to deal with the information produced in the consumption process of customers is very important, and also the timely collection of valuable information can provide scientific guidance for the improvement of business management in the future. In this paper, the main applications and daily management decision of hotel data center are analyzed in depth.

\section{The characteristics of data center}

For the definition of data center, there has been no a very clear concept classification yet in professional studies. From the perspective of hardware, data center is a data processing area composed by a set of facilities; from the perspective of hardware, it can be seen as a data warehouse with a large capacity. For the business management of a hotel, using high-configuration computer for establishing data center is a reform trend of information-based hotel management, and using computer to aid manual data processing is with the advantages of multiple aspects.

\section{Concentration}

Hotel is a management organization offering consumers with catering, accommodation, entertainment and other integrated services, and there are increasingly more service projects offered by hotels to consumers along with the continued increase of hotel service market demands, as shown in figure 1. Meanwhile, the amount of the information necessary to management personnel to process becomes much greater, and obviously the traditional manual data processing is unable to meet the requirements of hotel management. Data center possesses data storage function, and the hotel data center based on computer service model can intensively process all sorts of information and help the front-end and back-end personnel to implement centralized processing for internal information. 


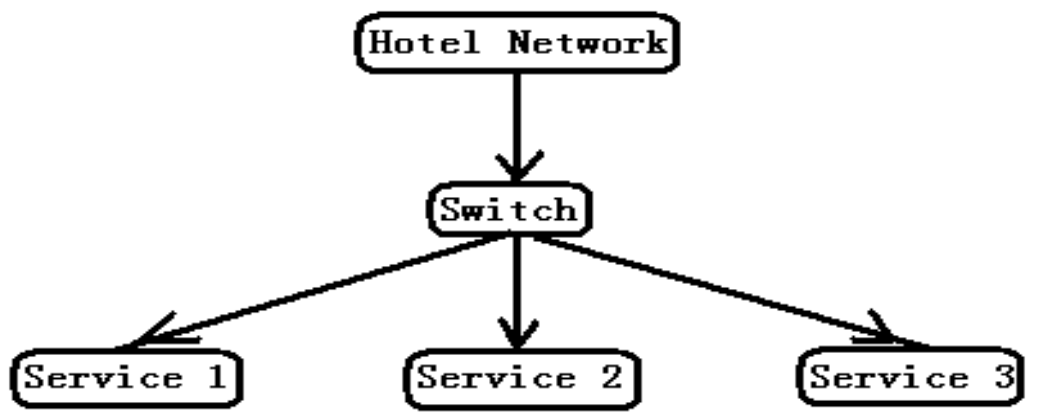

Figure 1: The hotel network model

\section{Targeted}

The requirements of different customers on hotel services are different, and this essentially depends on the economic strength and consumption level of consumers. To facilitate the development of integrated hotel management model, a personalized service model can be set up by choosing and matching data center facilities, so as to provide corresponding service projects for different customers and also coordinate the orderly implementation of the internal operation process. For example, hotels offer the consumer services of different grades according to the groups of different incomes, and also enter the information in data center, so as to facilitate the information query and search of personnel and also improve the quality of customer services.

\section{Science and technology}

The introduction of data center is a development product of hotel management oriented at science and Technology, and this means that high technologies are being integrated into the business plan of the hotel industry. In hotels, computer-majored hardware facilities have been equipped, and also an office network conforming to internal business management has been established so that automatic control and management can be implemented for all sorts of data within hotels. For example, the application functions of data center can provide a diversified operation platform for hotel personnel so that the difficulties of information processing can be controlled, the deployment of all information can be quickly completed in a short time, and the convenient and orderly operation is maintained.

\section{The main application functions of hotel data center}

Data center is a set of complex facilities. It includes not only computer system and other supporting devices such as communication and storage system, and also redundant data communication connections, environmental control equipment, monitoring equipment and all sorts of safety devices. From the structure, it is seen that data center commonly consists of multiple hardware and software facilities, and it has played a key role in the processing of all sorts of information. Along with the promotion of diversified hotel business management model, the integration of information technology into hotel management is an inevitable decision made by business operators.

\section{Providing network services}

Data center and the Internet are mutually connected, which can provide necessary network services for hotel management. Generally, to apply the Internet access, only one internet-enabled PC plus data center software is required, so that the difficulty of hotel management center network is weakened. The access of a hotel to the Internet is implemented through router; to install a router mentioned in this paper, some settings are necessary to deploy in the router, and also a broadband access line can be directly connected to PC without ISP service and it is only necessary to choose and use suitable dynamic domain software according to acquired Internet IP address. A superior network 
operation environment not only makes it easy for the application of hotel management office network, and also provides a good Internet environment for consumers, as shown in figure 2.

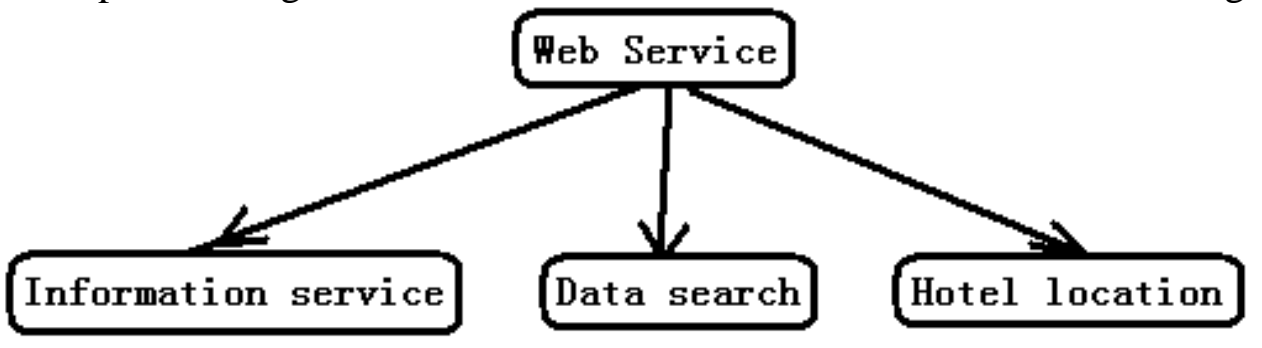

Figure 2: The network service function

\section{Implementing a uniform decision}

A unified measurement is implemented first for all sorts of market segments in Chinese Internet, enterprise services, and Internet users/audiences, and thus the unified researches on different market segments, different enterprise services, and different user audience groups are realized. This is a reflection to the application of data center to the innovation of hotel management. For some high-grade hotels, a data center is necessary to install for aiding its internal control so that the communication between service personnel and customers becomes convenient and also the best quality services are offered to consumers. In addition, the transverse, longitudinal, and point-to-point statistics and analysis and the reform and innovation in the function use between different network mediums are realized, and also a high-tech and diversified business system is created.

\section{Promoting technology innovation}

The development trend of enterprise-level data center is to possess high flexibility and adaptability. For example, the data center can make a rapid change according to the external demands and also speed up the technical reforms of hotel office management. The virtualization technology is taken as an example: the creation of the modular data center has been implemented by it, and also it is very suitable for hotel management control mode. At present, the rapidly changing business needs and the latest technologies with large energy consumption and refrigeration requirements are promoting the changes of data center, and all these mean that it is necessary for hotel management to take the road of technology innovation. Therefore, it can be seen that the optimization and transformation of data center play a promoting role in the application functions of hotel, and also symbolizes the arrival of a number of high-level hotel consumptions.

\section{The comprehensive decision for the daily management of data center}

On the one hand, data center is a centralized storage warehouse of hotel information, in which information is selectively collected in the early days according to the daily business requirements, and then can be called timely in the later use. Thus, the shortcomings of the traditional hotel management model are changed. On the other hand, data center is an operation dependent on the Internet data transmission, and the network is a basic platform of hotel work. From the perspective of the computer application technology development course, the network often suffers many potential risks in the data transmission process and especially the security risks of the data in the wireless communication technology are very high. Therefore, the daily management should be strengthened during the period of establishing data center, and the key is to prevent the adverse impacts caused by the loss of information.

\section{Security management}

In terms of the small-range wireless communication transmission, there is a great risk for information to be stolen in transmission, and the commercial information transmission is especially 
easy to be eavesdropped and stolen by illegal personnel. The sound signal transmission is taken as an example: encrypted processing is not made to the sound information transmitted by hotels, so the information can be acquired by cracking the codes in the actual transmission, and then a great amount of confidential business information is stolen and also a huge security risk is caused for business management. To solve this problem, it is necessary for a hotel to do a good security management for network data transmission. For example, the information review in the early transmission can be strengthened, and the transmission should be intercepted once abnormal conditions are found; the monitoring for the use of network data can be done well so that the abnormal data infected by viruses can be timely screened out.

\section{Equipment management}

It is an inevitable choice for a hotel to introduce a data center for aiding business management, but it is also necessary to carry out a detailed study on the potential hidden troubles of the data center when recognizing the functions of the information-based management mode. In data transmission, there are security threats from multiple aspects, and also it is possible to result in the loss of information if software and hardware equipment is damaged. When the computer hardware system is implementing tasks, the data operation process will be interrupted after failure occurs, and simultaneously the unprocessed information is likely to lose. Therefore, in the selection of software and hardware equipment, it is necessary for a hotel to start from the demands of the daily operation and management and study out a corresponding equipment management plan. Besides, an automatic data monitoring platform can be set up so that a secured and stable environment can be provided for the operations of the data center.

\section{Conclusion}

In short, data center is a data warehouse established for all information, and setting it in a hotel can give full play to the superior data processing functions and also makes it easy for the popularization and application of the information-based management mode. Considering the potential risks of computer network transmission, it is necessary for a hotel to regularly update the original data management mode and make an advanced solution for the daily management. Through the optimization of the daily management decision-making, the application of the data center to hotel management operation can be accelerated.

\section{Acknowledgement}

The author thanks the Wuhan Tourism Administration, Hubei, China, Tourism Research Grant \# WL2014C010, for providing support.

\section{References}

[1] Summit Forum about the Construction of Data Center Computer Room. Discussion on the Highly-reliable System Design for the Computer Room of Data Center [J]. Electrical Technology of Intelligent Buildings, 2011 (05).

[2] Wei Gao. Discussion on the Design of the Comprehensive Wiring System in Intelligent Light-current System [J]. Small and Medium-sized Enterprise Management and Technology, 2011(11).

[3] Haishu Wang, Xiaofeng Zhao. The Implementation Scheme for Low-cost and Long-distance Ethernet Transfer [J]. Information System Engineering, 2011(11).

[4] Bin Shen. Study on the Construction Plan of Network Integrated Wiring Training Room [J]. The Modern Reading (Education Edition), 2011(19). 